\title{
Cognitive consequences of timeframe bias. On the link between working memory, cognitive switching, and time perspective
}

\author{
Joanna Witowska ${ }^{1}$ (D) $\cdot$ Marcin Zajenkowski ${ }^{1}$ \\ Published online: 25 May 2019 \\ (C) The Author(s) 2019
}

\begin{abstract}
The present study examined how time perspective is associated with working memory updating and cognitive switching. Additionally, stress states and mood as potential mediators of the relationship between time perspective and cognitive performance were analysed. During two sessions participants $(n=200)$ completed a set of questionnaires measuring time perspective, task-related stress states, and mood. Moreover, in two separate sessions they performed working memory updating and switching tasks. The results indicated that two time perspectives, i.e. Present Fatalism and Past Positive, were associated with updating. Furthermore, mediation analysis showed that positive mood accounted for these relationships. Specifically, Present Fatalism was correlated with low positive mood and in turn, worse working memory scores, whereas Past Positive was associated with high positive mood leading to better performance on the working memory task. None of the time perspective dimensions correlated with cognitive switching. These findings shed more light on the cognitive consequences of timeframe bias and suggest new approaches in research on time perspective and cognitive functioning.
\end{abstract}

Keywords Time perspective $\cdot$ Working memory $\cdot$ Updating $\cdot$ Switching

\section{Introduction}

Cognitive aspects of psychological time have been widely studied in various contexts, such as the perception of the passage of time and the estimation of duration (Matthews and Meck 2016; Wearden et al. 2014). Recently, with growing interest time perspective has come to be understood more as an aspect of personality (Zimbardo and Boyd 1999) and a link to cognitive functioning has been observed (Witowska and Zajenkowski 2018; Zajenkowski et al. 2015, 2016a, b). One of the important findings in this area is the association between certain time perspective dimensions and executive control (Witowska and Zajenkowski 2018; Zajenkowski et al. 2016b). However, previous studies have focused solely on inhibition, while executive control is a broader construct (Diamond 2013). The aim of the current study is to examine

Joanna Witowska

joanna.witowska@psych.uw.edu.pl

Marcin Zajenkowski

zajenkowski@psych.uw.edu.pl

1 Faculty of Psychology, University of Warsaw, Stawki 5/7, 00-183 Warsaw, Poland further the relationship between time perspective and executive functioning. Specifically, we were interested in working memory (updating) and switching.

\section{Time Perspective}

The concept of time perspective (TP), originally proposed by Zimbardo and Boyd (1999), has recently been explored in a number of studies and contexts (see Kostić and Chadee 2017; Stolarski et al. 2015). Time perspective has been defined as an 'often nonconscious process whereby the continual flow of personal and social experiences is assigned to temporal categories, or time frames, that help to give order, coherence, and meaning to those events' (Zimbardo and Boyd 1999, p. 1271). In other words, our thoughts, emotions and experiences are usually located in the past, the present or the future. For instance, when we recall a memory, we focus on the past; when we form an expectation, we concentrate on the future. Although TP has been defined as a process, people may manifest a tendency to concentrate on a particular time horizon and thus TP may be treated as a disposition (Zimbardo and Boyd 1999). In its original conceptualization (Zimbardo and Boyd 1999) and subsequent developments of TP theory (Carelli et al. 2011), six time perspectives have been distinguished: Past Positive, Past Negative, Present Hedonism, Present 
Fatalistic, Future Negative, and Future Positive. Each TP is associated with specific psychological correlates and real-life outcomes (Kostić and Chadee 2017; Stolarski et al. 2015). Past Positive is characterized by positive perception and acceptance of past events, sentimentality, and attachment to traditions and rituals (Zimbardo and Boyd 1999). In contrast, Past Negative TP reflects a negative and antagonistic view of past events. Present Hedonism is associated with a tendency to concentrate on pleasant activities and immediate gratification without thinking about future consequences. Present Fatalism reflects the belief that life is ruled by luck and faith and is therefore hopeless, unpredictable, and unstable. Future Positive encompasses the motivation to achieve goals and success, whereas Future Negative reflects worry and anxiety about future events and a perception of the future as a threat.

According to TP theory (Zimbardo and Boyd 1999), individuals tend to automatically concentrate on specific time perspectives. However, researchers have identified an ideal time perspective profile, i.e. balanced time perspective (BTP) that is regarded as the most adaptive (Boniwell and Zimbardo 2004). Balanced time perspective is understood as the ability to switch between time dimensions in response to the requirements and demands of the environment (Boniwell and Zimbardo 2004).

The theoretical characteristics of TP suggests its potential connection with cognitive functioning (Boniwell and Zimbardo 2004; Zimbardo and Boyd 1999). Indeed, it has already been shown that some TPs, as well as BTP, rely on cognitive resources such as fluid intelligence and executive control (Witowska and Zajenkowski 2018; Zajenkowski et al. 2016a, b). The general conclusion from these investigations is that two TP dimensions are systematically linked to poorer cognitive performance: Past Negative and Present Fatalism. These TPs were found to be negatively associated with fluid intelligence (Zajenkowski et al. 2016a) as well as tasks measuring cognitive inhibition (Witowska and Zajenkowski 2018). Moreover, these studies revealed that specific state experiences associated with cognitive performance mediated these relationships. Specifically, Present Fatalism was linked to low task engagement, Past Negative to high distress in the context of cognitive task performance (Witowska and Zajenkowski 2018; Zajenkowski et al. 2016a). Furthermore, BTP was correlated with high levels of fluid intelligence and inhibition (Zajenkowski et al. 2016a, b). It has been suggested that fluid ability and inhibitory control allow individuals to be less biased toward a particular time zone, especially maladaptive ones such as Past Negative and Present Fatalism (Zajenkowski et al. 2016b).

Previous studies have shown executive control to be a crucial factor in some TPs. However, in these studies executive control was examined only in the context of cognitive inhibition. Executive control is a wide construct encompassing both working memory and switching. As we show below, the latter two functions might also be relevant for the processes underlying TP, i.e. assigning experience to a particular time frame.

\section{Updating and Switching and their Non-cognitive Correlates}

Executive control is defined as the ability to override automatic reactions and achieve adopted goals (Diamond 2013). In the most popular approach, executive control comprises three related but discrete processes: inhibition, switching, and working memory updating (Friedman and Miyake 2017; Miyake et al. 2000). These processes are believed to be helpful in challenging everyday problems, adapting to novel situations, and resisting temptation (Unger and Karbach 2017). Indeed, executive control is a strong predictor of a number of real-life outcomes, e.g. weight loss (Lawrence et al. 2015), academic achievement (Best et al. 2011), quality of life (Brown and Landgraf 2010; Davis et al. 2010), job success (Bailey 2007), and even the success of soccer players (Vestberg et al. 2012).

In the current investigation we were interested in two executive functions, i.e. working memory (WM) updating and switching. Updating is an ability that allows one to actively hold and mentally manipulate information in one's working memory while shielding it from distraction (Baddeley 2007). In other words, it allows maintenance of relevant information, while removing irrelevant details (Morris and Jones 1990). Working memory updating plays a crucial role in reordering important and unimportant items and connecting different ideas or facts (Diamond 2013). Updating involves the temporal sequencing and monitoring of information, tagging it as either old or new, important or unimportant, depending on the current situation (Miyake et al. 2000). In research on working memory, two kinds of tasks are mainly used, i.e. the span task and the $n$-back task. The crucial point is that these two types of tasks are weakly correlated and probably reflect different processes of WM (Redick and Lindsey 2013). Span tasks are typically used to measure working memory capacity and require information to be kept in mind in the face of distraction (Unsworth and Engle 2005), whereas n-back tasks capture working memory updating and require the constant refreshing of information (Kane et al. 2007). In the current study, the process of updating is investigated as part of the executive control concept (Miyake et al. 2000).

Switching is defined as the ability to switch between tasks or mental sets, the cognitive flexibility necessary in tasks with quickly changing rules or requiring different perspectives to be taken (Miyake et al. 2000). Measures of switching typically present participants with three situations: a task (A) with one instruction, followed by a task (B) with another instruction, followed by a task with two instructions (switching between $\mathrm{A}$ and B). In the switching task, two types of costs can be measured: first, mixing costs, which reflect the difference between 
performance (accuracy and time reaction) in a pure block (one task only) and a mixed block (switching between tasks); and second, switching costs, which reflect the difference between non-switch trials and switch trials within a mixed block (see, Philipp et al. 2008; Rogers and Monsell 1995).

Updating and switching have been linked to personality traits (Avisar and Shalev 2011; Jensen-Campbell et al. 2002), as well as affect (Gabel and McAuley 2018; Martin and Kerns 2011; Walters and Hines-Martin 2018). For instance, in a review of studies on mood and executive control, Mitchell and Phillips (2007) concluded that positive mood increases performance in switching/cognitive flexibility tasks but only in situations where new information is presented, whereas working memory can be impaired by positive mood. They also concluded that negative affect has less influence on executive functions. However, recent empirical studies concerning affective states and these executive functions provide rather ambiguous findings. For example, in several studies high negative affect weakened working memory updating ( $n$-back tasks; Brose et al. 2012; Gray 2001; Shackman et al. 2006). Moreover, there is also evidence that specific affect, i.e. anxiety, impairs WM capacity (span task; Derakshan and Eysenck 1998; Eysenck et al. 2005). Furthermore, Riediger et al. (2011) showed that the association between everyday mood and working memory capacity may be curvilinear. Specifically, they found that low-intensity positive and negative affect slightly enhanced working memory, while high-intensity positive and negative affect impaired working memory performance particularly when both positive and negative affect were high.

Research findings also show confusion with respect to positive mood and WM. For instance, Martin and Kerns (2011) found that positive mood (experimentally induced) impaired WM capacity, whereas other studies have indicated that positive affect improves WM (n-back, Gray 2001; span task, Yang et al. 2013). Other researchers have suggested that dopamine level may be responsible (mediate) for the beneficial role of positive mood on WM performance (Ashby et al. 1999); specifically, that dopamine increases positive affect and that its projection into the prefrontal cortex facilitates working memory.

Studies of task switching and mood are rather rare. Generally, they consistently show positive affect to improve cognitive flexibility (Isen 2008; Yang and Yang 2014). For instance, the study conducted by Yang and Yang (2014) indicated that induced positive affect was beneficial for switching costs but not mixing costs. The finding that positive mood lowers switching costs was further supported by an experimental study using neuroscience methods and a dopamine explanation, as in the case of WM (Wang et al. 2017).

Although the aforementioned findings and literature reviews (e.g. Walters and Hines-Martin 2018) show inconsistencies across studies, one can attempt to draw out some general conclusions on the links between updating, switching, and affect. First, most studies of WM show that negative mood has no effect or hinders WM, whereas positive mood enhances it. Likewise, in the case of switching, positive mood enhances cognitive flexibility whereas negative mood has no effect or impairs it (Dreisbach and Goschke 2004; Wang et al. 2017). The lack of clear conclusions from studies of mood and executive control may be associated with the fact that the studies reviewed used different conceptualizations of WM (updating or capacity) and different study designs (everyday experienced vs. experimentally induced mood). Another possibility is that positive and negative mood are not extremes of a continuum, but rather independent factors influencing cognitive performance simultaneously. For instance, a person could be not very happy, but, at the same time, not necessarily be sad. Similar proposition has been put forward in relation to motivation and affect (Maio and Esses 2001). Specifically, it has been found that approach and avoidance motivation to affect are at least somewhat distinct.

\section{The Current Study}

In the current study we aimed to further examine associations between executive control and TP. Specifically, we focused on the link between TP and updating and switching, as well as the role that affective states may play in this relationship. We believe that there are theoretical and empirical reasons to link $\mathrm{TP}$ with updating and switching. Time perspective seems to require cognitive resources associated with memory updating, e.g. establishing mental representations of events and encoding them in the specified time frame, as well as switching, i.e. activating an adequate time perspective in response to changing conditions (Boniwell and Zimbardo 2004; Zimbardo and Boyd 1999). Moreover, on the basis of previous research on time perspective, cognitive processes, and their correlates, below we postulate more specific associations between TPs, balanced TP, and updating and switching.

First, we expected that two time perspectives, Past Negative and Present Fatalism, would be associated with lower WM updating and switching (H1). Previous studies have shown that these two TPs are systematically correlated with poorer cognitive functioning (Witowska and Zajenkowski 2018; Zajenkowski et al. 2016a, 2016b). Both TPs are strongly associated with negative emotionality, e.g. anxiety, neuroticism, and negative mood (Stolarski et al. 2014; Zimbardo and Boyd 1999), which may weaken task performance (Derakshan and Eysenck 1998; Gray 2001; Shackman et al. 2006). Thus we expected that negative mood would mediate the link between these TPs and updating and switching (H2).

However, a deeper analysis of the relationship between Past Negative, Present Fatalism, and cognition has revealed some differences between these TPs. Specifically, in some 
investigations the multi-dimensional model of subjective stress state related to cognitive performance proposed by Matthews et al. (2002) was used. In this model, three broad factors are distinguished: task engagement (interest in a task, high energy, motivation, and concentration), distress (tension, negative mood, and lack of confidence), and worry (negative thoughts, self-focused attention, low self-esteem, and cognitive interference). It has been found that Past Negative is correlated with high distress, which mediates its association with fluid intelligence and executive control (Witowska and Zajenkowski 2018; Zajenkowski et al. 2016a). In the case of Present Fatalism it is task engagement (low) and worry that mediate the cognitive performance of fatalists. Given these associations, we expected that distress would mediate the link between Past Negative TP and executive control (updating and switching; H3), whereas worry and task engagement would mediate Present Fatalism's association with executive control (H4).

Past Negative and Present Fatalism aside, previous studies have found no correlation between the remaining TPs and cognition (Witowska and Zajenkowski 2018; Zajenkowski et al. 2016a). It should be acknowledged, though, that Past Positive and Future Positive have been linked to higher levels of positive affect (Stolarski et al. 2014) and lower stress states in response to cognitive performance (Witowska and Zajenkowski 2018).

Finally, switching and WM updating seem to defy the characteristics of balanced TP. Balanced time perspective has been defined as "the mental ability to switch effectively between TPs depending on task features, situational considerations, and personal resources, rather than be biased toward a specific TP that is not adaptive across situations" (Zimbardo and Boyd 1999, p.1285). It is possible that domain-specific "temporal switching" may rely on general switching resources. Furthermore, it has previously been suggested that WM plays a crucial role in activating the controlled system as opposed to the automatic system (e.g. Barrett et al. 2004), which may lead to the more appropriate rather than biased activation of a specific TP. Indeed, previous studies suggest that balanced TP may be associated with higher cognitive abilities. Specifically, balanced TP has been found to be correlated with higher inhibition and fluid intelligence (Zajenkowski et al. 2016a, b). Moreover, the states experienced during performance mediated these relationships: more balanced individuals experienced lower stress while solving cognitive tests, which in turn resulted in their higher scores. Taking into account the theoretical and empirical characteristics of BTP, we hypothesized that BTP would be associated with higher executive control including working memory updating and switching (H5). Furthermore, we expected that (low) stress states would mediate these relationships (H6).
In the current study we tested the associations between time perspective, WM updating, and cognitive switching. Additionally, we controlled for state responses to these tasks, as previous research has indicated that they may play an important role in the TP-cognition link. Specifically, we assessed participants' task-related stress states as postulated in the aforementioned Matthews et al.'s (2002) model, i.e. task engagement, distress, and worry. Moreover, we measured mood experienced before the task using a three-dimensional model distinguishing energetic arousal (contrasting vigor with fatigue), tense arousal (nervousness vs. relaxation), and hedonic tone (pleasantness vs. unhappiness; Matthews et al. 1990).

\section{Method}

\section{Participants}

A total of 200 participants took part in the study (100 females and 100 males). One (female) participant was excluded for failing to complete the study. The mean age of the sample was 22.80 years $(\mathrm{SD}=3.78)$ with a range $18-40$ years. The majority of the sample were Polish undergraduate students ( $71 \%$ secondary education degree, $29 \%$ higher degree), recruited by four experimenters mainly via social media. Written informed consent was obtained from all participants, who were informed about the procedure, data confidentiality, anonymity, and gratification.

\section{Procedure}

The study was conducted over two sessions, with at least a 24$\mathrm{h}$ break, in a quiet laboratory at the University of University of Warsaw in the presence of an experimenter. During each session participants completed a set of questionnaires and performed computerized tasks. Each session took the following order: first, participants completed the mood (UMACL) and pre-task stress (DSSQ) questionnaires; then, they performed the cognitive tasks ( $n$-back vs set switching; the order being rotated between subjects); and finally, they completed the post-task stress (DSSQ) and remaining questionnaires.

\section{Measures}

Time perspective was measured using the Zimbardo Time Perspective Inventory (ZTPI) as amended by Carelli et al. (2011) in the Polish version validated by Jochemczyk et al. (2017). This inventory has 64 items covering six different time dimensions: Past-Negative $(\alpha=.80)$; Past-Positive $(\alpha=.78)$; Present-Hedonistic $(\alpha=.81)$; Present-Fatalistic $(\alpha=.70)$; Future-Positive $(\alpha=.81)$; and, Future-Negative $(\alpha=.63)$. 
The Future-Negative subscale was added to the original ZTPI scale. Participants responded on a five-point scale (from 1 "strongly disagree" to 5 - "strongly agree"). Based on the scores from the six TPs, balanced time perspective was calculated using the following formula:

$$
\begin{gathered}
\sqrt{ }(\mathrm{oPN}-\mathrm{ePN})^{2}+(\mathrm{oPP}-\mathrm{ePP})^{2}+(\mathrm{oPF}-\mathrm{ePF})^{2} \\
+(\mathrm{oPH}-\mathrm{ePH})^{2}+(\mathrm{oF}-\mathrm{eF})^{2}+(\mathrm{oFN}-\mathrm{eFN})^{2}
\end{gathered}
$$

where $\mathrm{o}=$ observed and $\mathrm{e}=$ expected score; $\mathrm{ePN}=1.95$, $\mathrm{ePP}=4.60, \mathrm{ePF}=1.50, \mathrm{ePH}=3.90, \mathrm{eF}=4.00, \mathrm{eFN}=1.8$ (Rönnlund et al. 2017). The outcome measure is the deviation from balanced time perspective (DBTP), where the lower the DBTP score the more balanced the TP profile.

Mood was measured using the mood adjective check list (UMACL; Matthews et al. 1990) in the Polish version (Zajenkowski and Matthews 2019). The scale has 24 items (adjectives) divided into three subscales: Energetic Arousal (EA; measuring positive affect and energy); Tense Arousal (TA; negative affect and tension); and Hedonic Tone (HT; pleasant, contented mood, high energy, low tension) which are calculated basing on key scores. Respondents described their current mood on a four-point Likert scale (from 1 "definitely yes" to 4 - "definitely no"). Cronbach's alphas for mood scales measured before updating task were: $\alpha=85$ for EA; $\alpha=.63$ for TA, and $\alpha=.90$ for HT. Cronbach's alphas before switching task were $\alpha=.81$ (EA); $\alpha=.53$ (TA); $\alpha=.89$ (HT).

Stress states were measured with the Dundee Stress State Questionnaire (DSSQ; Matthews et al. 2002),specifically the short version (see Matthews and Zeidner 2012) translated into Polish (Zajenkowski and Zajenkowska 2015). The DSSQ measures three factors: task engagement, distress, and worry. It has 24 items with five-point response scales (from 0 "definitely disagree" to 4 - "definitely agree"). The internal consistency of the Polish version is high (Zajenkowski and Zajenkowska 2015), with Cronbach's alphas ranging from .75 to .84 .

Updating was measured using a 2-back task in two versions verbal (containing numbers), and visual (containing figures) adapted from Chuderski and Nęcka (2012). Stimuli in the tasks were digits or figures, each approximately 2.5 by $2.5 \mathrm{~cm}$ in size and presented for 1000 milliseconds (ms) following a $600 \mathrm{~ms}$ blank screen. In each task (numbers and figures), a total of 162 stimuli were presented serially to participants in three sessions of 54 stimuli. Between sessions, participant took a short break. Each session included sixteen 2-back target repetitions of stimuli. Participants were instructed to respond to repetitions and suppress responses to all other items. The score on the tasks was the number of correctly detected repetitions. The variables "updating figure" and "updating number" are indicators of accuracy in the two updating tasks. We also computed the principal component factor of both indicators ("updating factor").

Switching was measured with two tasks using different materials: verbal (numbers) and visual (figures). The computerized tasks created for this study were similar to those used by Karbach and Kray (2009) and Nęcka et al. (2012), and consisted of three blocks: two single tasks and one mixed block. In the task with number stimuli, in the first block (40 trials) participants were asked to categorize numbers as either smaller (press ' $Z$ ') or bigger (press ' $M$ ') than five. In the second block (40 trials), participants categorized digits as odd (press ' $\mathrm{Z}$ ') or even (press ' $\mathrm{M}$ '). The digit "5" was removed from the set of stimuli. Each trial in both blocks had the following procedure: first, a fixation cross \# appeared for $1000 \mathrm{~ms}$, followed by a target stimulus presented for $500 \mathrm{~ms}$. In the last, mixed, block participants were asked to switch between the two previous tasks according to a cue - the single word "EVEN?" or "SMALLER?"- presented every two trials, the cues indicating which instruction participants were to follow. The mixed block consisted of 80 randomly presented trials with the following procedure: first, a fixation cross appeared $(1000 \mathrm{~ms})$, followed by a cue $(500 \mathrm{~ms})$, followed in turn by a target stimulus ( $3000 \mathrm{~ms})$ and a blank screen $(500 \mathrm{~ms})$. There was a short practice session consisting of four trials before the mixed block.

In the version with figures, in the first block participants were asked to categorize figures as either small or big. In the second block, the task was to categorize whether a figure was sharp or smooth. In the mixed block, participants had to switch between the two tasks. The cues were a single word:"SHAPE?" or "SIZE?" The procedure, duration of display, and number of trials were the same as in the number version of the task. We calculated mixing costs and register accuracy in the mixed series in both tasks. We treated the mean difference between accuracy in the single blocks (mean of the two single blocks) and mixed blocks as an indicator of mixing costs (Philipp et al. 2008). We also used an indicator of mean accuracy in the mixed block.

\section{Results}

\section{Time Perspective and Working Memory Updating}

Table 1 presents the correlations between all the variables. The results indicate that two time perspectives were associated with updating: Past Positive and Present Fatalism. Specifically, Past Positive was associated with higher scores on the $n$-back task, whereas the Present Fatalistic dimension 


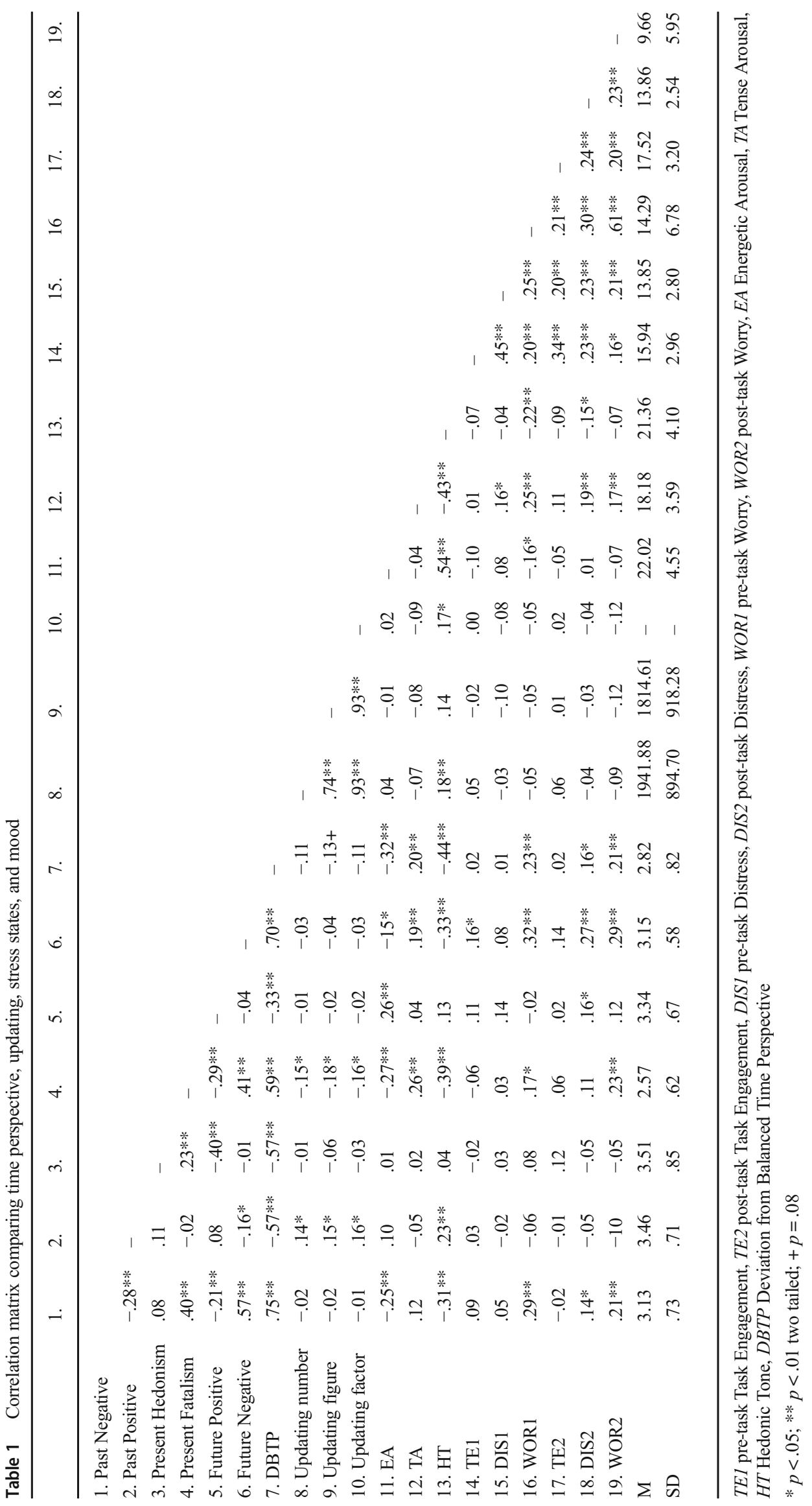


Table 2 Regression analyses with pre-task stress states distress and time perspective as predictors and post-task stress scores for distress as dependent variables in updating

\begin{tabular}{|c|c|c|c|c|c|c|c|c|}
\hline & & & Outcome: Post-t & $\mathrm{k}$ distre & & & & \\
\hline & $\Delta R 2$ & $\beta$ & & $\Delta R 2$ & $\beta$ & & $\Delta R 2$ & $\beta$ \\
\hline Step 1 & .02 & & & .02 & & & .02 & \\
\hline Sex & & .08 & Sex & & .08 & Sex & & .08 \\
\hline Age & & .09 & Age & & .09 & Age & & .09 \\
\hline Step 2 & $.05 * *$ & & & $.05 * *$ & & & $.05 * *$ & \\
\hline Sex & & .06 & Sex & & .06 & Sex & & .06 \\
\hline Age & & .10 & Age & & .10 & Age & & .10 \\
\hline DIS1 & & $.23 * *$ & DIS1 & & $.23 * *$ & DIS1 & & $.23 * *$ \\
\hline Step 3 & $.02 *$ & & & .01 & & & $.07 * *$ & \\
\hline Sex & & .07 & Sex & & .07 & Sex & & .10 \\
\hline Age & & .11 & Age & & .08 & Age & & .12 \\
\hline DIS1 & & $.23^{* *}$ & DIS1 & & $.22 * *$ & DIS1 & & $.21 * *$ \\
\hline Past Negative & & $.15^{*}$ & Future Positive & & .12 & Future Negative & & $.27 * *$ \\
\hline
\end{tabular}

DIS1 pre-task Distress. $* p<.05 ; * * p<.01$ was adversely related to updating. These time perspectives correlated with both versions of the updating tasks as well as the updating factor. Deviation from balanced time perspective was weakly negatively correlated with the updating figure task with approaching significance $(p<.08)$. Because of the large number of variables, we additionally calculated Bonferroni correction for the correlations by dividing the alpha level by the number of comparisons of each variable. This analysis revealed that correlations between Past Positive dimension and updating task (number, figure and factor) are not significant. It appeared for Present Fatalism and its correlations with updating as well. The associations between n-back number task and hedonic tone is not significant. Moreover, all correlations which are marked with'*' in the table are not significant anymore. We also analyzed state responses to the $n$-back task. In order to test how the level of stress changed in all participants while taking the task, within-subjects $t$-tests were performed. The analysis indicated that the level of task engagement increased (before: $\mathrm{M}=15.94, \mathrm{SD}=2.96$; after: $\mathrm{M}=17.52, \mathrm{SD}=3.20, p<.001$ ), whereas worry decreased (before: $\mathrm{M}=14.29, \mathrm{SD}=6.78$; after: $\mathrm{M}=9.66, \mathrm{SD}=5.95, \mathrm{p}$ $<.001)$ after the task.

Analysis of mood and stress responses to the $n$-back task revealed that Past Negative TP was negatively correlated with hedonic tone and energetic arousal, and positively with worry (pre- and post-task) and distress (post-task). Past Positive was

Table 3 Regression analyses with pre-task stress states worry and time perspective as predictors and post-task stress scores for worry as dependent variables in updating task

\begin{tabular}{|c|c|c|c|c|c|c|c|c|c|c|c|}
\hline & & & Outcome: Post & sk worr & & & & & & & \\
\hline & $\Delta R 2$ & $\beta$ & & $\Delta R 2$ & $\beta$ & & $\Delta R 2$ & $\beta$ & & $\Delta R 2$ & $\beta$ \\
\hline Step 1 & .00 & & & .00 & & & .00 & & & .00 & \\
\hline Sex & & .04 & Sex & & .04 & Sex & & .04 & Sex & & .04 \\
\hline Age & & .04 & Age & & .04 & Age & & .04 & Age & & .04 \\
\hline Step 2 & $.38 * *$ & & & $.38 * *$ & & & $.38 * *$ & & & $.38 * *$ & \\
\hline Sex & & -.00 & Sex & & -.00 & Sex & & -.00 & Sex & & .11 \\
\hline Age & & .11 & Age & & .11 & Age & & .11 & Age & & -.01 \\
\hline WOR1 & & $.62 * *$ & WOR1 & & $.62 * *$ & WOR1 & & $.62 * *$ & WOR1 & & $.62 * *$ \\
\hline Step 3 & $.02 *$ & & & .00 & & & .01 & & & .01 & \\
\hline Sex & & .01 & Sex & & -.00 & Sex & & .01 & Sex & & .11 \\
\hline Age & & .11 & Age & & .11 & Age & & .11 & Age & & -.01 \\
\hline WOR1 & & $.60 * *$ & WOR1 & & $.61 * *$ & WOR1 & & $.59 * *$ & WOR1 & & $.61 * *$ \\
\hline Present Fatalism & & $.14 *$ & Past Negative & & .04 & Future Negative & & .11 & DBTP & & .08 \\
\hline
\end{tabular}

WORl pre-task Worry. $* p<.05 ; * * p<.01$ 


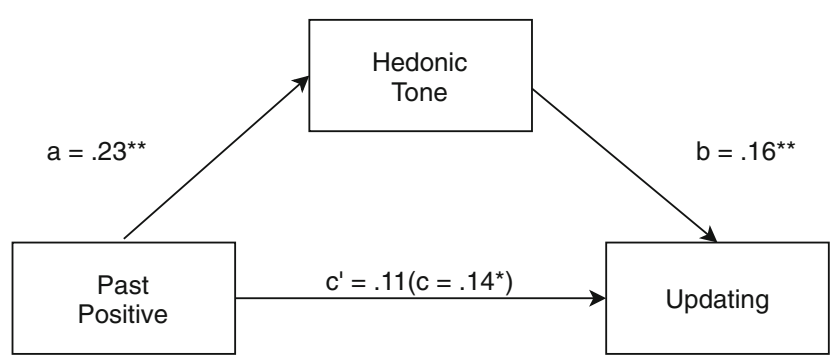

Fig. 1 Relationships between Past Positive, hedonic tone, and updating task The paths with a's and b's are direct, $\mathrm{c}$ is the total effect from Past Positive to updating task and c' is the direct path from Past Positive to updating task, controlling for hedonic tone. ${ }^{*} \mathrm{p}<0.05 ; * * p<0.01$

associated with high hedonic tone. Present Fatalism was negatively correlated with hedonic tone and energetic arousal, and positively with tense arousal. It was also associated with measurements of worry. The Future Positive dimension was positively associated with energetic arousal and post-task distress, while Future Negative was negatively associated with energetic arousal and hedonic tone, and positively with tense arousal. It was also positively correlated with pre-task task engagement and worry, and post-task distress and worry. Deviation from balanced time perspective was negatively correlated with energetic arousal and hedonic tone, and positively with tense arousal, both states of worry, and post-task distress.

On the basis of the associations between time perspectives and stress states, we conducted a series of regression models investigating whether time perspectives predict post-task stress states, controlling for baseline states (Tables 2 and 3). The post-task score is more representative of the state during task performance, while the pre-task score represents general dispositions and anticipation of the task (e.g. Matthews and Zeidner 2012; Zajenkowski and Matthews 2019). In each regression model we introduced sex and age in step 1, a baseline stress state in step 2, and a TP dimension in step 3. The results demonstrated that after controlling for baseline state, Past Negative TP was a positive predictor of distress, while Present Fatalism positively predicted worry.

The results presented above reveal that Past Positive and Present Fatalism were associated with hedonic tone and

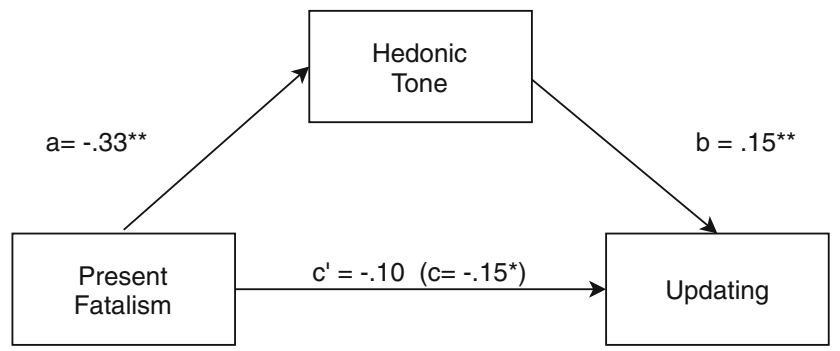

Fig. 2 Relationships between Present Fatalism, hedonic tone, and updating task The paths with a's and b's are direct, $\mathrm{c}$ is the total effect from Present Fatalism to updating task and c' is the direct path from Present Fatalism to updating task, controlling for hedonic tone. $* p<0.05 ; * * p<0.01$ updating. Moreover, updating (number, $n$-back) and hedonic tone were positively related as well. Therefore, we went on to examine whether hedonic tone mediated the link between these time perspectives (separately for Past Positive and Present Fatalism) and updating (number, $n$-back). We used the PROCESS macro for SPSS developed by Hayes (2015) to test for indirect effects by calculating confidence intervals (CIs) with 5000 bootstraps. The first analysis indicated that the total effect of Past Positive TP on updating $(\beta=.14$; $p<.01$ ) was smaller upon inclusion of the mediator (hedonic tone) and the direct effect was not significant $(\beta=.11, p=.13$; see Fig. 1), while the indirect effect was significant .04, $p<0.05,95 \% \mathrm{CI}=[.006 ; .088]$. In the second analysis, the total effect of Present Fatalistic TP on updating ( $\beta=-.15 ; \mathrm{p}$ $<.01$ ) was smaller upon inclusion of the mediator (hedonic tone) and the direct effect was not significant $(\beta=-.10$, $p=.19$; see Fig. 2), whereas the indirect effect was significant $-.05, \mathrm{p}<0.05,95 \% \mathrm{CI}=[-.107 ;-.003]$. These analyses reveal that positive mood mediated the associations between two time perspectives (Past Positive and Present Fatalism) and updating (number, $n$-back task).

\section{Time Perspective and Switching}

Table 4 presents the correlations between switching, TP, and state measures. Most importantly, none of the TP dimensions correlated with switching, although some of them displayed associations with stress states and mood. Specifically, Past Negative and Present Fatalism were negatively correlated with energetic arousal and hedonic tone, and positively correlated with tense arousal. Furthermore, both TPs were positively associated with pre- and post-task worry, while fatalism was also correlated with post-task distress. The Past Positive perspective was associated with higher energetic arousal and hedonic tone, while Future Positive displayed a positive association with energetic arousal. The Future Negative perspective showed the same correlation pattern as that of the Past Negative TP. We additionally calculated Bonferroni correction for the correlations by dividing the alpha level by the number of comparisons of each variable. This analysis revealed that Past Negative is not significantly correlated with pos-task worry, Past Positive is not correlated with energetic arousal and Present Fatalism is not associated with post-task distress. Moreover, all correlations which are marked with'*' in the table are not significant anymore.

In order to test how the level of stress changed in participants while performing the switching task, within-subjects $t$ tests were performed. We found that the level of task engagement after performance $(\mathrm{M}=17.7$; $\mathrm{SD}=3.33)$ was higher than that before $(\mathrm{M}=15.61 ; \mathrm{SD}=2.84, p<.001)$, while worry decreased (before: $\mathrm{M}=14.46, \mathrm{SD}=6.42$; after: $\mathrm{M}=9.63$, $\mathrm{SD}=6.31, \mathrm{p}<.001)$. This pattern of changes is the same as that in the session with WM updating. 


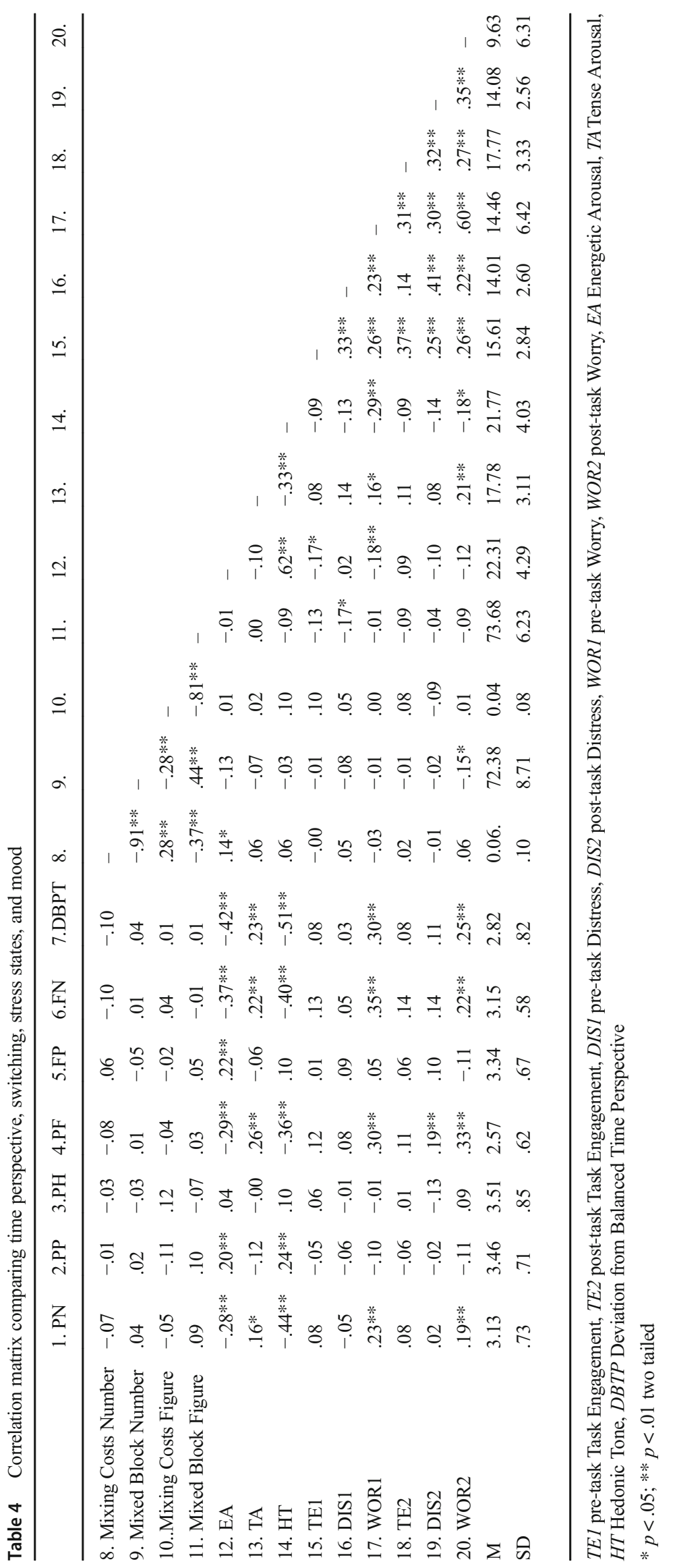


Table 5 Regression analyses (betas) with pretask stress states distress and time perspective as predictors and post-task stress scores for distress as dependent variables in switching task

\begin{tabular}{|c|c|c|}
\hline \multicolumn{2}{|c|}{ Outcome: Post-task distress } & \multirow[b]{2}{*}{$\beta$} \\
\hline & $\Delta R 2$ & \\
\hline Step 1 & .03 & \\
\hline Sex & & .12 \\
\hline Age & & .13 \\
\hline Step 2 & $.15^{* *}$ & \\
\hline Sex & & .09 \\
\hline Age & & .07 \\
\hline DIS1 & & $.40^{* *}$ \\
\hline Step 3 & $.03 * *$ & \\
\hline Sex & & .10 \\
\hline Age & & .10 \\
\hline DIS1 & & $.38 * *$ \\
\hline Present Fatalism & & $.17 * *$ \\
\hline
\end{tabular}

Subsequently, we conducted a series of regressions investigating whether time perspectives predict post-task stress states, controlling for the baseline of particular stress states (Tables 5 and 6). In each case we introduced sex and age in step 1, the first measurement of a given stress state in step 2, and one TP dimension in step 3. The analyses indicated that Present Fatalism was a positive predictor of post-task distress as well as worry, when controlling for the baseline levels of these stress states.

What is worth pointing out too is that there were significant $(p<.05)$ correlations between mixing costs in the switching tasks and updating, ranging from -.16 to -.24 . Correlations between accuracy in the mixed series of the switching tasks ranged from .28 to $.35(\mathrm{p}<.05)$.

\section{Discussion}

The primary aim of the current study was examine associations between time perspective and executive control operationalized as WM updating and switching. We found that two time perspectives, i.e. Past Positive and Present Fatalism, were significantly associated with WM updating whereas switching showed no correlations with TPs. Furthermore, experienced states were taken into account as potential mediators of the TP-cognition link. We found that participants' positive mood (hedonic tone) mediated the relationships between Present Fatalism and updating as well as Past Positive and updating. Finally, some of the TPs predicted stress responses to cognitive tasks. Below we discuss these findings in detail.

In the current study, Present Fatalism was negatively correlated with the WM updating task. This result is consistent with previous studies showing that Present Fatalism impairs various aspects of cognitive functioning, including fluid and crystallized intelligence (Zajenkowski et al. 2016a) as well as cognitive inhibition (Witowska and Zajenkowski 2018). Moreover, in a recent study Rönnlund and Carelli (2018) found that among TPs, Present Fatalism was the strongest correlate of general cognitive ability among older adults (60-90 years old) and that this TP was significantly predicted by their intelligence score at age 18 . There could be several explanations for the poorer cognitive functioning of fatalistic

Table 6 Regression analyses with pre-task stress states worry and time perspective as predictors and post-task stress scores for worry as dependent variables in switching task

\begin{tabular}{|c|c|c|c|c|c|c|c|c|c|c|c|}
\hline & & Outco & Post-task worry & & & & & & & & \\
\hline & $\Delta R 2$ & $\beta$ & & $\Delta R 2$ & $\beta$ & & $\Delta R 2$ & $\beta$ & & $\Delta R 2$ & $\beta$ \\
\hline Step 1 & .00 & & & .00 & & & .00 & & & .00 & \\
\hline Sex & & .04 & Sex & & .04 & Sex & & .04 & Sex & & .04 \\
\hline Age & & -.02 & Age & & -.02 & Age & & -.02 & Age & & -.02 \\
\hline Step 2 & $.36 * *$ & & & $.36 * *$ & & & $.36 * *$ & & & $.36^{* *}$ & \\
\hline Sex & & .09 & Sex & & .09 & Sex & & .09 & Sex & & .09 \\
\hline Age & & -.01 & Age & & -.01 & Age & & -.01 & Age & & -.01 \\
\hline WOR1 & & $.61 * *$ & WOR1 & & $.61 * *$ & WOR1 & & $.61 * *$ & WOR1 & & $.61 * *$ \\
\hline Step 3 & .00 & & & $.02 * *$ & & & .00 & & & .01 & \\
\hline Sex & & .09 & Sex & & .09 & Sex & & .09 & Sex & & .09 \\
\hline Age & & -.01 & Age & & .00 & Age & & -.01 & Age & & -.01 \\
\hline WOR1 & & $.59 * *$ & WOR1 & & $.56^{* *}$ & WOR1 & & $.60 * *$ & WOR1 & & $.58 * *$ \\
\hline Past Negative & & .06 & Present Fatalism & & $.16^{* *}$ & Future Negative & & .02 & DBTP & & .07 \\
\hline
\end{tabular}

WORl pre-task Worry. $* p<.05 ; * * p<.01$ 
individuals. Theoretical characteristics of Present Fatalism highlight a specific thinking style and belief system, or cognitive aspects, associated with this TP. Fatalism has been defined as the belief that life is unpredictable and that one has little control over external events; this attitude results in hopelessness and helplessness and the belief that the present must be borne with resignation because humans are at the mercy of whimsical "fate" (Zimbardo and Boyd 1999). These views may have consequences for many areas of life, including cognitive functioning. As some authors have suggested, when faced with a challenging intellectual test fatalists may experience a lack of motivation because they do not believe that they can overcome the difficulties in order to succeed in the task (Zajenkowski et al. 2016a). The findings concerning fatalists' affective reactions and self-views seem to be in line with this interpretation. First, Zajenkowski et al. (2016a) found that present fatalists perceived their cognitive ability to be low, and that this perceived intelligence mediated the association between fatalism and actual intelligence. These authors suggested that fatalists' negative views of their intelligence may undermine their confidence and result in poorer performance. Second, across studies Present Fatalism is associated with a state response to cognitive performance. Specifically, it has been found that fatalism is correlated with greater worry and low task engagement, and that these stress states mediate its link with performance (Witowska and Zajenkowski 2018; Zajenkowski et al. 2016a). Interestingly, in both sessions of the current study present Fatalism was associated with high worry experienced during the task. The state of worry reflects negative thoughts about one's performance, self-focused attention, and cognitive interference. These task-unrelated thoughts may be a reaction to the task demands encountered. Finally, the current research also revealed that negative mood may, to some extent, be responsible for the poorer cognitive functioning in Present Fatalistic TP.

Interestingly, in the present study we found that Past Positive TP was positively associated with WM updating and that high positive mood mediated this relationship. This result seems to be in line with previous findings indicating that Past Positive is strongly associated with various aspects of autobiographical memory, including a strong sense of recollection, vivid, emotional recall, clarity of visual-spatial imagery, and recall of more positive emotions (Ely and Mercurio 2010). Additionally, other studies have suggested that working memory plays an important role in successful emotion regulation following instruction (Schmeichel et al. 2008). It has been also shown that people with a high level of WM engage more effectively in spontaneous emotion regulation following negative feedback, compared with those with lower WM (Schmeichel and Demaree 2010). Specifically, Schmeichel and Demaree (2010) found experimentally that high levels of WM predicted greater self-enhancement and less negative affect following negative feedback about participants' emotional intelligence. Taking all these findings into account, one might question whether individuals with a high level of working memory perceive and experience life events more positively and in turn develop positive perceptions of the past, i.e. high Past Positive. It should be acknowledged, however, that this interpretation is speculative and the causal relation between WM and Past Positive should be examined in future studies.

Balanced time perspective showed no significant associations with switching and WM updating. It should be acknowledged, however, that the latter correlation reached tendency level $(p=0.08)$ while the magnitude of the coefficient $(\mathrm{r}=.13)$ was similar to that obtained in an inhibition task in a study by Witowska and Zajenkowski (2018) on a larger sample. Nevertheless, the weak or null relations with WM and switching shed some light on the nature of BTP. As mentioned above, Zimbardo and Boyd (1999) emphasized that efficient switching between perspectives is a key component of BTP. Our results suggest that these mechanisms probably do not rely on the cognitive ability to switch between tasks, perhaps because of one important conceptual problem-BTP is typically measured as a static construct, i.e. a profile or configuration of traits. Unfortunately, there are no methods to assess more dynamic and processual aspects of BTP, that is, how people switch between time zones in response to situational demands. However, it is still possible that BTP may be associated with cognitive switching if we could find a way of measuring its processual facet.

\section{Limitations and Future Studies}

Although the current study sheds some light on the relationships between time perspective and cognition, it has also a few limitations. Most importantly, our research was correlation in nature, which leaves the problem of causality unresolved. Hypothetically, one may assume that executive control is more primary and determines the development of specific timeframe bias. However, further, longitudinal, studies are needed to shed more light on the causal direction of the TPcognition link. Another problem is that we did not include a screening procedure in order to exclude over psychopathology, however, in the study advertisement this requirement of participation (physical and mental health) was literally noted. Moreover, our sample (students) was quite homogeneous in terms of demographic variables and it would be useful to test other groups in future studies.

It would be valuable to examine other cognitive processes and tasks in relation to time perspective. In the current study we used a task measuring working memory updating in two versions: one using numbers and the other using figures. However, in other studies different content (e.g. letters) as well as scoring procedure (e.g. based on reaction time) was used (e.g. Artuso and Palladino 2018). Another interesting option 
would be to use domain-specific stimuli associated with time (e.g. words) instead of content-free stimuli of numbers or letters. Additionally, so far only higher order cognitive functions (e.g. intelligence, executive control) have been analyzed in the context of TP. It would also be interesting to examine more basic processes, such as short-term memory and prospective memory, and their relation to habitual patterns of categorizing past, present, and future events.

\section{Conclusion}

The present study has given some answers to questions concerning TPs and cognitive functioning relationships. Specifically, we found that Present Fatalism was associated with poorer performance on a working memory updating task, whereas Past Positive time perspective correlated with better performance on this task. Moreover, individuals scoring high on Present Fatalism experienced more negative mood, while individuals with high Past Positive TP experienced more positive mood. Mood level mediated the relationships between both TPs and cognitive performance. Further investigations should focus on resolving the question of causality in these relationships and seeking a new operationalization of BTP.

Acknowledgements The work was supported from a grant no 2016/23/ N/HS6/02928 funded by National Science Centre in Poland.

\section{Compliance with Ethical Standards}

Conflict of Interest The Authors declare that there is no conflict of interest.

Ethical Approval Research Ethic Committee of Faculty of Psychology, University of Warsaw.

Open Access This article is distributed under the terms of the Creative Commons Attribution 4.0 International License (http:// creativecommons.org/licenses/by/4.0/), which permits unrestricted use, distribution, and reproduction in any medium, provided you give appropriate credit to the original author(s) and the source, provide a link to the Creative Commons license, and indicate if changes were made.

\section{References}

Artuso, C., \& Palladino, P. (2018). The role of memory updating in shallow native and opaque second language learning. Second Language Research, 1-20. https://doi.org/10.1177/ 0267658318777022.

Ashby, F. G., Isen, A. M., \& Turken, A. U. (1999). A neuropsychological theory of positive affect and its influence on cognition. Psychological Review, 106, 529-550. https://doi.org/10.1037/ 0033-295X.106.3.529.

Avisar, A., \& Shalev, L. (2011). Sustained attention and behavioral characteristics associated with ADHD in adults. Applied
Neuropsychology, 18(2), 107-116. https://doi.org/10.1080/ 09084282.2010 .547777 .

Baddeley, A. D. (2007). Working memory, thought, and action. Oxford: Oxford University Press. https://doi.org/10.1093/acprof:oso/ 9780198528012.001.0001.

Bailey, C. E. (2007). Cognitive accuracy and intelligent executive function in the brain and in business. Annals of the New York Academy of Sciences, 1118, 122-141. https://doi.org/10.1196/annals.1412.011.

Barrett, L. F., Tugade, M. M., \& Engle, R. W. (2004). Individual differences in working memory capacity and dual-process theories of the mind. Psychological Bulletin, 130, 553-573. https://doi.org/10. 1037/0033-2909.130.4.553.

Best, J. R., Miller, P. H., \& Naglieric, J. A. (2011). Relations between executive function and academic achievement from ages 5 to 17 in a large, representative national sample. Learning and Individual Differences, 21(4), 327-336. https://doi.org/10.1016/j.lindif.2011. 01.007 .

Boniwell, I., \& Zimbardo, P. G. (2004). Balancing time perspective in pursuit of optimal functioning. In P. A. Linley \& S. Joseph (Eds.), Positive psychology in practice (pp. 165-178). Hoboken: Wiley. https://doi.org/10.1002/9780470939338.ch10.

Brose, A., Schmiedek, F., Lövdén, M., \& Lindenberger, U. (2012). Daily variability in working memory is coupled with negative affect: The role of attention and motivation. Emotion, 12, 605-617. https://doi. org/10.1037/a0024436.

Brown, T. E., \& Landgraf, J. M. (2010). Improvements in executive function correlate with enhanced performance and functioning and health-related quality of life: Evidence from 2 large, double-blind, randomized, placebo-controlled trials in ADHD. Postgraduate Medicine, 122, 42-51. https://doi.org/10.3810/pgm.2010.09.2200.

Carelli, M. G., Wiberg, B., \& Wiberg, M. (2011). Development and construct validation of the Swedish Zimbardo time perspective inventory. European Journal of Psychological Assessment, 27, 220 227. https://doi.org/10.1027/1015-5759/a000076.

Chuderski, A., \& Nęcka, E. (2012). The contribution of working memory to fluid intelligence: Capacity, control, or both? Journal of Experimental Psychology. Learning, Memory, and Cognition, 38, 1689-1710. https://doi.org/10.1515/ppb-2016-0003.

Davis, J. C., Marra, C. A., Najafzadeh, M., \& Lui-Ambrose, T. (2010). The independent contribution of executive functions to health related quality of life in older women. BMC Geriatrics, 10, 16-23. https://doi.org/10.1186/1471-2318-10-16.

Derakshan, N., \& Eysenck, M. W. (1998). Working memory capacity in high trait-anxious and repressor groups. Cognition \& Emotion, 12, 697-713. https://doi.org/10.1080/026999398379501.

Diamond, A. (2013). Executive functions. Annual Review of Psychology, 64, 135-168. https://doi.org/10.1146/annurev-psych-113011143750.

Dreisbach, G., \& Goschke, T. (2004). How positive affect modulates cognitive control: Reduced perseveration at the cost of increased distractibility. Journal of Experimental Psychology. Learning, Memory, and Cognition, 30(2), 343-353. https://doi.org/10.1037/ 0278-7393.30.2.343

Ely, R., \& Mercurio, A. (2010). Time perspective and autobiographical memory: Individual and gender differences in experiencing time and remembering the past. Time \& Society, 20(3), 375-400. https://doi. org/10.1177/0961463X10366081.

Eysenck, M. W., Payne, S., \& Derakshan, N. (2005). Trait anxiety, visuospatial processing, and working memory. Cognition and Emotion, 19(8), 1214-1228. https://doi.org/10.1080/02699930500260245.

Friedman, N. P., \& Miyake, A. (2017). Unity and diversity of executive functions: Individual differences as a window on cognitive structure. Cortex, 86, 186-204. https://doi.org/10.1016/j.cortex.2016.04.023.

Gabel, M. S., \& McAuley, T. (2018). Does mood help or hinder executive functions? Reactivity may be the key. Personality and Individual 
Differences, 128, 94-99. https://doi.org/10.1016/j.paid.2018.02. 027.

Gray, J. (2001). Emotional modulation of cognitive control: Approach withdrawal states double dissociate spatial from verbal two-back task performance. Journal of Experimental Psychology: General, 130, 436-452. https://doi.org/10.1037/0096-3445.130.3.436.

Hayes, A. F. (2015). An index and test of linear moderated mediation. Multivariate Behavioral Research, 50, 1-22. https://doi.org/10. 1080/00273171.2014.962683.

Isen, A. M. (2008). Some ways in which positive affect influences decision making and problem solving. In M. Lewis, J. Haviland-Jones, \& L. F. Barrett (Eds.), Handbook of emotions (3rd ed., pp. 548-573). New York: Guilford Press. https://doi.org/10.1017/ CBO9780511609978.013.

Jensen-Campbell, L. A., Rosselli, M., Workman, K. A., Santisi, M., Rios, J. D., \& Bojan, D. (2002). Agreeableness, conscientiousness, and effortful control processes. Journal of Research in Personality, 36, 476-489. https://doi.org/10.1016/S0092-6566(02)00004-1.

Jochemczyk, Ł., Pietrzak, J., Buczkowski, R., Stolarski, M., \& Markiewicz, Ł. (2017). You only live once: Present-hedonistic time perspective predicts risk propensity. Personality and Individual Differences, 115, 148-153. https://doi.org/10.1016/j.paid.2016.03. 010.

Kane, M. J., Conway, A. R. A., Hambrick, D. Z., \& Engle, R. W. (2007). Variation in working memory capacity as variation in executive attention and control. In A. R. A. Conway, C. Jarrold, M. J. Kane, A. Miyake, \& J. N. Towse (Eds.), Variation in working memory (pp. 21-48). New York: Oxford University Press. https://doi.org/10. 1093/acprof:oso/9780195168648.003.0002.

Karbach, J., \& Kray, J. (2009). How useful is executive control training? Age differences in near and far transfer of task-switching training. Developmental Science, 12, 978-990. https://doi.org/10.1111/j. 1467-7687.2009.00846.x.

Kostić, A., \& Chadee, D. (Eds.). (2017). Time perspective. Theory and practice. Palgrave Macmillan. https://doi.org/10.1057/978-1-13760191-9.

Lawrence, N. S., O'Sullivan, J., Parslow, D., Javaid, M., Adams, R. C., Chambers, C. D., Kos, K., \& Verbruggen, F. (2015). Training response inhibition to food is associated with weight loss and reduced energy intake. Appetite, 95, 17-28. https://doi.org/10.1016/j.appet. 2015.06.009.

Maio, G. R., \& Esses, V. M. (2001). The need for affect: Individual differences in the motivation to approach or avoid emotions. Journal of Personality, 69(4), 583-615. https://doi.org/10.1111/ 1467-6494.694156.

Martin, E. A., \& Kerns, J. G. (2011). The influence of positive mood on different aspects of cognitive control. Cognition and Emotion, 25(2), 265-279. https://doi.org/10.1080/02699931.2010.491652.

Matthews, W. J., \& Meck, W. H. (2016). Temporal cognition: Connecting subjective time to perception, attention, and memory. Psychological Bulletin, 142(8), 865-907. https://doi.org/10.1037/bul0000045.

Matthews, G., \& Zeidner, M. (2012). Individual differences in attentional networks: Trait and state correlates of the ANT. Personality and Individual Differences, 53, 574-579. https://doi.org/10.1016/j.paid. 2012.04.034.

Matthews, G., Jones, D. M., \& Chamberlain, A. G. (1990). Refining the measurement of mood: The UWIST mood adjective checklist. British Journal of Psychology, 81, 17-42. https://doi.org/10.1111/j. 2044-8295.1990.tb02343.x.

Matthews, G., Campbell, S. E., Falconer, S., Joyner, L., Huggins, J., \& Gilliland, K. (2002). Fundamental dimensions of subjective state in performance settings: Task engagement, distress and worry. Emotion, 2, 315-340. https://doi.org/10.1037/1528-3542.2.4.315.

Mitchell, R. L. C., \& Phillips, L. H. (2007). The psychological, neurochemical and functional neuroanatomical mediators of the effects of positive and negative mood on executive functions.
Neuropsychologia, 45(4), 617-629. https://doi.org/10.1016/j. neuropsychologia.2006.06.030.

Miyake, A., Friedman, N. P., Emerson, M. J., Witzki, A. H., Howerter, A., \& Wager, T. D. (2000). The unity and diversity of executive functions and their contributions to complex frontal lobe tasks: A latent variable analysis. Cognitive Psychology, 41(1), 49-100. https://doi. org/10.1006/cogp.1999.0734.

Morris, N., \& Jones, D. M. (1990). Memory updating in working memory: The role of central executive. British Journal of Psychology, 81, 111-121. https://doi.org/10.1111/j.2044-8295.1990.tb02349.x.

Nęcka, E., Lech, B., Sobczyk, N., \& Śmieja, M. (2012). How much do we know about our own cognitive control? Self-report and performance measures of executive functions. European Journal of Psychological Assessment, 28, 240-247. https://doi.org/10.1027/ 1015-5759/a000147.

Philipp, A. M., Kalinich, C., Koch, I., \& Schubotz, R. I. (2008). Mixing costs and switch costs when switching stimulus dimensions in serial predictions. Psychological Research, 72, 405-414. https://doi.org/ 10.1007/s00426-008-0150-x.

Redick, T. S., \& Lindsey, D. R. (2013). Complex span and $n$-back measures of working memory: A meta-analysis. Psychonomic Bulletin \& Review, 20, 1102-1113. https://doi.org/10.3758/s13423-0130453-9.

Riediger, M., Wrzus, C., Schmiedek, F., Wagner, G. G., \& Lindenberger, U. (2011). Is seeking bad mood cognitively demanding? Contrahedonic orientation and working-memory capacity in everyday life. Emotion, 11, 656-665. https://doi.org/10.1037/a0022756.

Rogers, R. D., \& Monsell, S. (1995). Costs of a predictable switch between simple cognitive tasks. Journal of Experimental Psychology: General, 124, 207-231. https://doi.org/10.1037/0096-3445.124.2. 207.

Rönnlund, M., \& Carelli, M. (2018). Deviations from a balanced time perspective in late adulthood: Associations with current $\mathrm{g}$ and $\mathrm{g}$ in youth. Intelligence, 71, 8-16. https://doi.org/10.1016/j.intell.2018. 09.002 .

Rönnlund, M., Äström, E., \& Carelli, M. (2017). Time perspective in late adulthood: Aging patterns in past, present and future dimensions, deviations from balance, and associations with subjective well-being. Timing and Time Perception, 5(1), 77-98. https://doi.org/10. 1163/22134468-00002081.

Schmeichel, B. J., \& Demaree, H. A. (2010). Working memory capacity and spontaneous emotion regulation: High capacity predicts selfenhancement in response to negative feedback. Emotion, 10, 739 744. https://doi.org/10.1037/a0019355.

Schmeichel, B. J., Volokhov, R., \& Demaree, H. A. (2008). Working memory capacity and the self-regulation of emotional expression and experience. Journal of Personality and Social Psychology, 95, 1526-1540. https://doi.org/10.1037/a0013345.

Shackman, A. J., Sarinopoulos, I., Maxwell, J. S., Pizzagalli, D. A., Lavric, A., \& Davidson, R. J. (2006). Anxiety selectively disrupts visuospatial working memory. Emotion, 6, 40-61. https://doi.org/ 10.1037/1528-3542.6.1.40.

Stolarski, M., Matthews, G., Postek, S., Zimbardo, P. G., \& Bitner, J. (2014). How we feel is a matter of time: Relationships between time perspectives and mood. Journal of Happiness Studies, 15(4), 809827. https://doi.org/10.1007/s10902-013-9450-y.

Stolarski, M., Fieulaine, N., \& van Beek, W. (2015). Time perspective theory: Review, research and application. Cham: Springer. https:// doi.org/10.1007/978-3-319-07368-2.

Unger, K., \& Karbach, J. (2017). Executive functions. In N. Pachana (Ed.), Encyclopedia of geropsychology (pp. 1-10). Singapore: Springer. https://doi.org/10.1007/978-981-287-082-7_275.

Unsworth, N., \& Engle, R. W. (2005). Individual differences in working memory capacity andlearning: Evidence from the serial reaction time task. Memory and Cognition, 33(2), 213-220. https://doi.org/ 10.3758/BF03195310. 
Vestberg, T., Gustafson, R., Maurex, L., Ingvar, M., \& Petrovic, P. (2012). Executive functions predict the success of top-soccer players. PLoS One, 7(4), e34731. https://doi.org/10.1371/journal.pone.0034731.

Walters, M., \& Hines-Martin, V. (2018). Overview of executive functions in mood and depressive disorders: A review of the literature. Archives of Psychiatric Nursing, 32(4), 617-637. https://doi.org/ 10.1016/j.apnu.2018.02.011.

Wang, Y., Chen, J., \& Yue, Z. (2017). Positive emotion facilitates cognitive flexibility: An fMRI study. Frontiers in Psychology, 8(1832). https://doi.org/10.3389/fpsyg.2017.01832.

Wearden, J., O'Donoghue, A., Ogden, R., \& Montgomery, C. (2014). Subjective duration in the laboratory and the world outside. In V. Arstillaand \& D. Lloyd (Eds.), Subjective time, the philosophy, psychology, and neuroscience of temporality (pp. 287-306). London: MIT Press. https://doi.org/10.7551/mitpress/8516.003.0022.

Witowska, J., \& Zajenkowski, M. (2018). How is perception of time associated with cognitive functioning? The relationship between time perspective and executive control. Time \& Society, 1-24. https://doi.org/10.1177/0961463X18763693.

Yang, H., \& Yang, S. (2014). Positive affect facilitates task switching in the dimensional change card sort task: Implications for the shifting aspect of executive function. Cognition and Emotion, 28(7), 1242 1254. https://doi.org/10.1080/02699931.2013.879053.

Yang, H., Yang, S., \& Isen, A. M. (2013). Positive affect improves working memory: Implications for controlled cognitive processing. Cognition and Emotion, 27(3), 474-482. https://doi.org/10.1080/ 02699931.2012 .713325

Zajenkowski, M., \& Matthews, G. (2019). Intellect and openness differentially predict affect: Perceived and objective cognitive ability contexts.
Personality and Individual Differences, 137, 1-8. https://doi.org/10. 1016/j.paid.2018.08.001.

Zajenkowski, M., \& Zajenkowska, A. (2015). Intelligence and aggression: The role of cognitive control and test related stress. Personality and Individual Differences, 81, 23-28. https://doi.org/10.1016/j. paid.2014.12.062.

Zajenkowski, M., Carelli, M. G., \& Ledzińska, M. (2015). Cognitive processes in time perspective. In M. Stolarski, N. Fieulaine, \& W. van Beek (Eds.), Time perspective theory; review, research and application (pp. 243-255). Cham: Springer. https://doi.org/10. 1007/978-3-319-07368-2_16.

Zajenkowski, M., Stolarski, M., Maciantowicz, O., Malesza, M., \& Witowska, J. (2016a). Time to be smart: Uncovering a complex interplay between intelligence and time perspectives. Intelligence, 58, 1-9. https://doi.org/10.1016/j.intell.2016.06.002.

Zajenkowski, M., Stolarski, M., Witowska, J., Maciantowicz, O., \& Łowicki, M. (2016b). Fluid intelligence as a mediator of the relationship between executive control and balanced time perspective. Frontiers in Psychology, 7(1844). https://doi.org/10.3389/fpsyg. 2016.01844.

Zimbardo, P. G., \& Boyd, J. N. (1999). Putting time in perspective: A valid, reliable individual-difference metric. Journal of Personality and Social Psychology, 77, 1271-1288 https://doi.org/10.1037/ 0022-3514.77.6.1271.

Publisher's note Springer Nature remains neutral with regard to jurisdictional claims in published maps and institutional affiliations. 